\title{
New Haptic Syringe Device for Virtual Angiography Training
}

\author{
Dongjin Huanga,*, Pengbin Tang ${ }^{\mathrm{b}}$, Xianglong Wang ${ }^{\mathrm{a}}$, Tao Ruan Wan ${ }^{\mathrm{c}}$, Wen Tang ${ }^{\mathrm{d}}$ \\ ${ }^{a}$ Shanghai Film Academy, Shanghai University, Shanghai 200072, China; \\ ${ }^{b}$ Département d'informatique et de recherche opérationnelle, Université de Montréal, Montréal, QC H3C3J7, Canada; \\ ${ }^{c}$ The Faculty of Science, Design and Technology, Pool House, University of Bournemouth, Poole BH12 5BB, UK; \\ ${ }^{d}$ The Faculty of Engineering and Informatics, University of Bradford, Bradford BD7 1DP, UK.
}

\section{A R T I C L E IN F O}

Article history:

Keywords: Virtual Angiography, Syringe Device, Haptic Feedback, Medical Training

\begin{abstract}
A B S T R A C T
Angiography is an important minimally invasive diagnostic procedure in endovascular interventions. Effective training for the procedure is expensive, time consuming and resource demanding. Realistic simulation has become a viable solution to addressing such challenges. However, much of previous work has been focused on software issues. In this paper, we present a novel hardware system-an interactive syringe device with haptics as an add-on hardware component to 3D VR angiography training simulator. Connected to a realistic 3D computer simulation environment, the hardware component provides injection haptic feedback effects for medical training. First we present the design of corresponding novel electronic units consisting of many design modules. Second we describe a curve fitting method to estimate injection dosage and injection speed of the contrast media based on voltage variation between the potentiometer to increase the realism of the simulated training. A stepper motor control method is developed to imitate the coronary pressure for force feedback of syringe. Experimental results show that the validity and feasibility of the new haptic syringe device for achieving good diffusion effects of contrast media in the simulation system. A user study experiment with medical doctors to assess the efficacy and realism of proposed simulator shows good outcomes.
\end{abstract}

(c) 2019 Elsevier B. V. All rights reserved.

\section{Introduction}

Cardiovascular diseases are the number one cause of death in the world [1]. Endovascular Intervention is an effective treatment for the diseases in modern medicine with advantages of limited hemorrhage, minimal wound, faster recovery and less complications compared with traditional open surgery. Angiography is an essential examination procedure in endovascular interventions to obtain clear medical images to identify lesion

\footnotetext{
${ }^{*}$ Corresponding author. E-mail addresses: djhuang@shu.edu.cn (D. Huang), pengbin.tang@umontreal.ca (P. Tang), 541771732@qq.com (X. Wang), wtang@bournemouth.ac.uk (T.R. Wan), t.wan@Bradford.ac.uk (W.Tang).
}

locations of tumors and peripheral vascular narrowing and assess cardiovascular damage [2]. Training for angiography and any minimally invasive endovascular interventions is resource demanding and time consuming. Virtual reality based training has become an effective means for medical training [3, 4]. When surgeons perform an angiography procedure, they would operate medical apparatus and instruments on patients, such as guidewires, catheters and syringe devices. Not only highfidelity computer simulation [5, 6, 7, 8] is important for computer based medical training, but also haptic feedback during the handling of the instruments [9, 10, 11] is crucial for the surgical training. Therefore, realistic simulations must include medical apparatus and instrument operations and handling.

The angiography operation starts from Seldinger technique, 


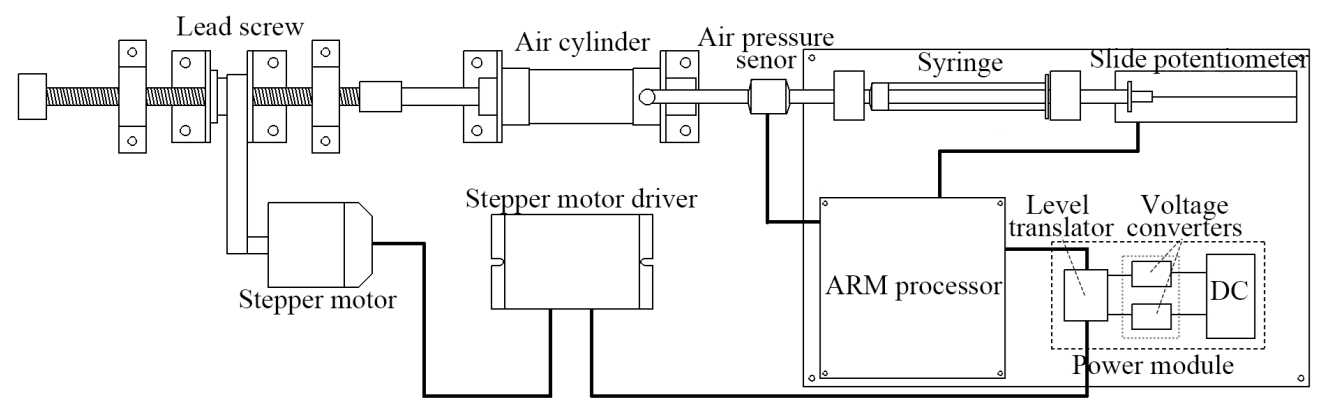

(a) The sketch of our hardware

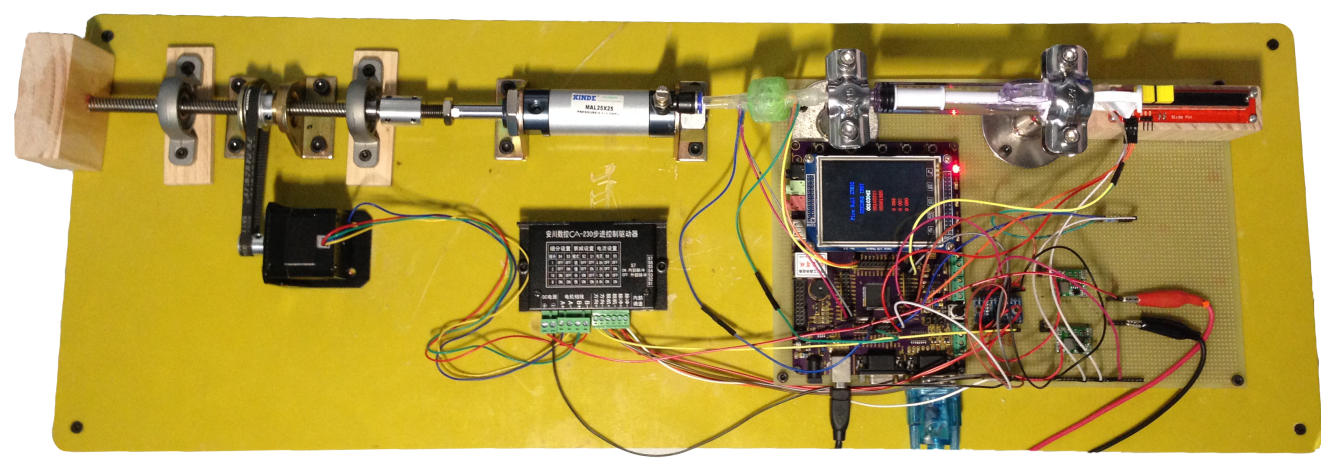

(b) The concrete model

Fig. 1. Syringe device

1 and surgeons can insert the catheter to the corresponding loca2 tion by introducing the guidewire to the lesions of blood vessel. 3 Finally, the contrast media can be injected into the blood ves4 sel by syringe via the catheter. After injection, the structure of blood vessel can be visualized under $\mathrm{x}$-ray, which makes surgeons clear the lesions' locations and status of blood vessel. At present, several angiography simulation software system$\mathrm{s}[12,13,14]$ have been presented. They were mainly focused on the modeling of guidewires and diffusion simulations of the contrast media in blood. Besides software system, one of the big challenges for virtual reality based training systems to be effective is the realism of the hardware system (e.g. haptics and physical feedbacks). Huang et al. [15] combined a Geomagic touch haptic device to steer guidewire for its navigation. Luboz et al. [16] have mainly focused on the training techniques instead of the simulation of angiography. They proposed a computer simulator for Seldinger technique, which included a simulated pulse to guide needle puncture palpation with haptics for the insertion of guidewires and catheters.

Contrast media injection by syringe is a crucial step for realistic training of angiography. For a realistic simulation, a simulator need to simulate the force feedback of syringe when injecting contrast media, due to the inner blood pressure. Moreover, the injection speed and dosage are also a very important part for the following diffusion simulation of contrast media in blood. However, above systems have largely ignored these factors. Although in [17] a syringe device was proposed to compute the dosage of contrast media by a constant injection speed, the method can not obtain accurate injection dosage and injec- tion speed for the contrast media. The trainees' handling of the instruments has a large influence on the injection speed and dosage, hence it is one of the most important aspects for effective angiography training. In dental diagnose, Poyade et al.[18] proposed a haptic training simulation for injection of anaesthesia into the region of the inferior alveolar nerve. In this system, they adopted the Geomagic Touch (formerly known as Phantom Omni) to control virtual needles, but only the button of the haptic device was utilized to administration the anaesthesia.

Validation of simulation system by medical doctors, who are the end users of the simulation system, are critical for any systems to be adopted in clinic practice. The commercial simulator "Vascular Interventional System Trainer(VIST)" has been evaluated by physicians [19, 20, 21, 22]. The simulator includes a mannequin, two monitors, joysticks for controlling fluoroscopy and a syringe for the injection of contrast media. A recent related patent [23] of this system has shown that the virtual contrast media was created by injecting air by a syringe without force feedback. Schuetz et al. [24] combined "CATHI-system", which firstly introduced by [25], with a full scale human patien$\mathrm{t}$ simulator and delivered simulator courses to medical professionals to validate the realism of the system. In this simulator, they used a real syringe for the contrast media injection with fluid instead of air, but unfortunately there is no force feedback within their device [26]. Commercial simulators are still far too expensive for many hospitals in everyday practice.

In this paper, we describe a novel hardware design using a real syringe device integrated with haptic feedback for angiography training. In this device, the force feedback of syringe can 


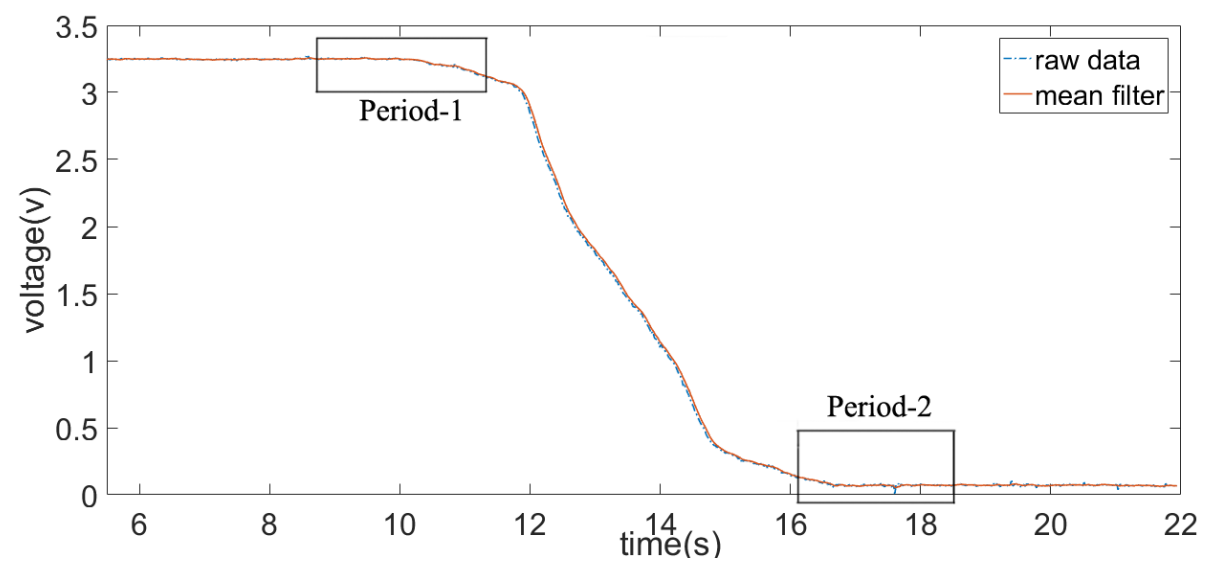

(a) Entire procedure

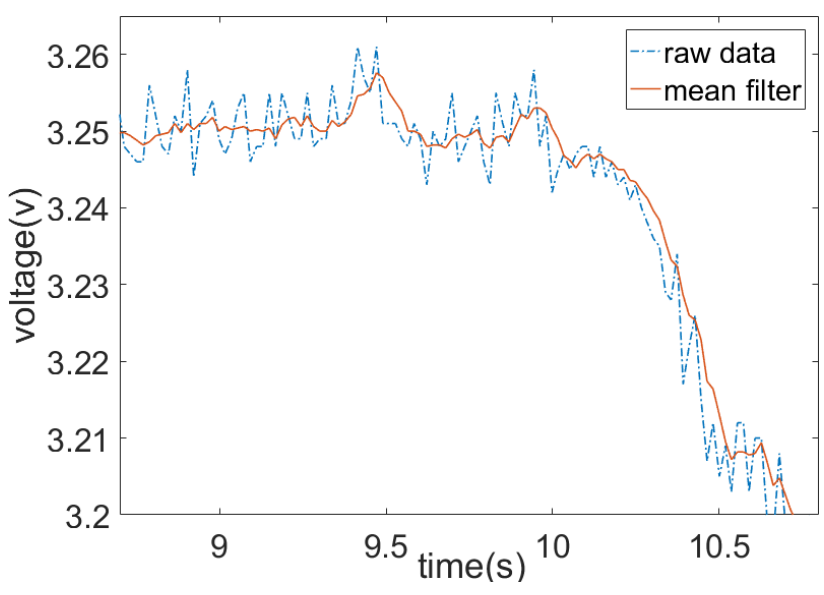

(b) Period-1

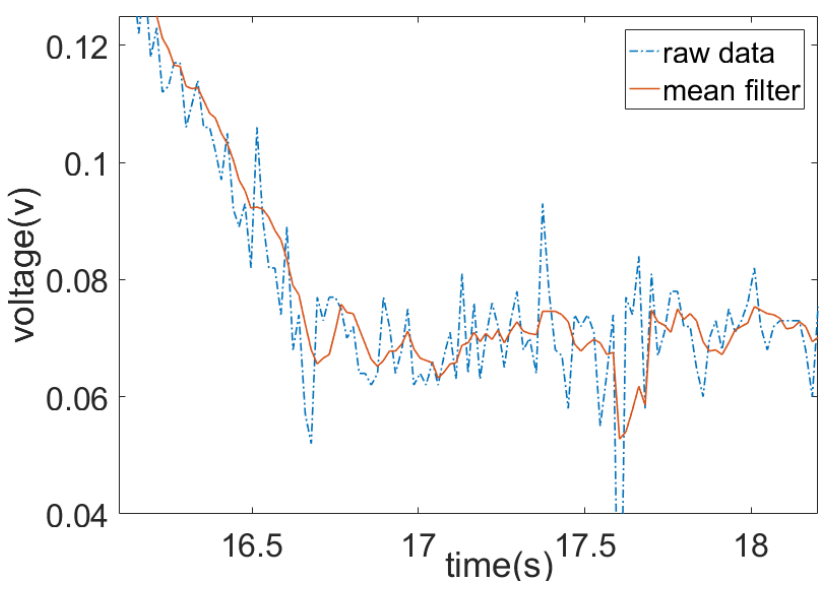

(c) Period-2

Fig. 2. Preprocess results comparison

be reached by simulating the inner blood pressure which will react to the piston of syringe. In addition, the injection speed and dosage will be obtained automatically when injecting contrast media. It is an add-on hardware component to our 3D virtual reality angiography training simulator to provide an integrated and realistic simulation environment.

\section{Design of A Haptic Syringe Device}

We describe the design of the interactive syringe device, including the method of step motor control and the curve fitting method for estimating injection dosages and the injection speed of the contrast media. The integration of the physical medical device with the simulation system is important to medical students to learn how to administrate the contrast media, which is the first step of initialisation of the virtual contrast media. The hardware system achieves effective injection handling with haptics feedback for medical training. We describe implementation details including different modules and experiment results, and evaluate how the proposed simulation system helps to improve the hand-eye coordination through both virtual and physical interactions by mimicking the real life procedural process and the hardware device for the medical procedure.

\subsection{Hardware design}

The surgeons will feel resistant force from the piston of syringe when they perform an angiography procedure due to the blood pressure. Since this is a main influence factor for the realistic training, the actuation main requirements for our hardware is to simulate this force feedback when trainees operating syringe and injecting contrast media into blood vessels. In addition, the hardware device also should automatically compute the injection speed and dosage for the initialization of angiography simulation.

Fig. 1 demonstrates the sketch of our proposed syringe device. The device includes a power module (which includes a level translator, voltage converters and a direct currency (DC)), a stepper motor module (which contains a stepper motor and its driver), a slide potentiometer, an air pressure sensor (BMP280 barometric pressure sensor from Bosch), a force sensing resistor (Flexiforce Sensor from TeKscan), an ARM processor, an air cylinder, a lead screw and a coronary control syringe normally used in the real medical operations.

The ADC interface of the ARM processor samples the voltage between the slide potentiometer. The piston of syringe is fixed with the slide potentiometer for injection together with the same distance. When moving the piston, the voltage of the slide potentiometer changes, thereby using the sampled voltage data 
to calculate the injection dosage and injection speed of contrast media.

The lead screw is adopted to convert the rotary motion of the stepper motor to a linear motion of screw. Therefore, we can use the stepper motor to control the piston of the air cylinder, meaning that we can control the inner pressure of the air cylinder by the stepper motor. And an air pressure sensor is adopted to sample the pressure inside this device.

\subsection{Injection status calculation}

\subsubsection{Data preprocess}

Due to the environment disturbing at interface of electronic components and sampling of ARM processor, sampled data exist some oscillates. It is, therefore, necessary to preprocess the raw data before estimating injection status for the contrast media. We compared median filter, mean filter and Kalman filter to de-noise the sampled data, and finally the recursive mean filter was chosen as the best one to preprocess the raw data. The processed results are show in Fig. 2, in which the dash-dot line represents the raw data and the right-pointing triangle denotes preprocessed data by recursive median filter. During this preprocess, the syringe begins with a static state, then the piston of the syringe is pushed to inject the contrast media (see Fig. 2(b)), finally, the piston is reaching at the end of the syringe (see Fig. 2(c)).

\subsubsection{Data fitting}

In order to compute the injection dosage and the injection speed of the contrast media while using the interactive syringe device, we propose a curve fitting method to further analyse the intrinsic characteristics of the pre-processed voltage data with regards to the injection status of the device. Firstly, we advance the piston to each dial on the syringe and record the ground truth of the volume data and the corresponding voltage data. Five groups of voltage-volume data are retrieved iteratively for accurate fitting.

Functions including Fourier, polynomial and a composite function are used to fit the scattered voltage-volume data. Finally, the composite function is selected as the best fitting curve:

$$
f(U)=\frac{4.968 U^{5}-34.77 U^{4}+77.02 U^{3}-81.3 U^{2}+97.75 U-5.823}{U^{4}-5.232 U^{3}+1.55 U^{2}+15.5 U+3.174}
$$

Finally, Equation(1) estimates the injection dosage from the sampled voltage data through the ARM processor in real-time. After getting the volume, the injection speed is obtained by the change of volume within a certain period of time:

$$
g(V)= \begin{cases}\frac{V_{\mathrm{t} \_ \text {min }}-V_{\text {cur }}}{\Delta t}, & V_{\text {cur }}<V_{\mathrm{t} \_ \text {min }} \\ 0, & V_{\text {cur }} \geq V_{\mathrm{t} \_ \text {min }}\end{cases}
$$

where $\Delta t$ represents the sampling time interval, $V_{\text {cur }}$ is the current volume, $V_{\mathrm{t} \_ \text {min }}$ is the minimum volume during an injection. In order to prevent current converted volume become lager than the previous converted volume, we use the minimum value of converted volume to update $V_{\mathrm{t} \_ \text {min }}$ continuously.

\subsection{Stepper motor control}

The force feedback from the syringe is mainly influenced by the inner blood pressure. The air cylinder is adopted to imitate the condition inside the blood vessel. Therefore, we employ a stepper motor to control the piston rod of the air cylinder to change the inner air pressure of the air cylinder, and a stepper motor control method to simulate the coronary pressure.

Firstly, we use several coronary blood pressure pulses [27] and for each pulse, several feature pressures are sampled with a number $n$ representing this pulse.

We randomly select a coronary pulse after the simulation end and compute the input number of pulses for the stepper motor control. This is because the operation of the stepper motor is controlled by the input frequency and the number of pulses, in order to simulate the given target coronary pressure $P_{\text {tar }}$ under the delta time $t_{\mathrm{d}}$, the target volume of air cylinder is computed:

$$
V_{\text {air_tar }}=V_{\text {air_total }}-\left(\frac{P_{\text {init }} V_{\text {total }}}{P_{\text {tar }}}-V_{\text {syr }}-V_{\text {ext }}\right)
$$

where $V_{\text {air total }}$ is the total volume of the air cylinder, $P_{\text {init }}$ is the pressure of the initial state, $V_{\text {total }}$ is the total volume in this device, $V_{\text {syr }}$ is the current syringe volume and $V_{\text {ext }}$ is the extra volume except the syringe and air cylinder, and then the pulses are used to drive the stepper motor to control the piston into the corresponding position according to $V_{\text {air_tar }}$. The target number of pulses is found:

$$
N_{\text {tar }}=\frac{V_{\text {air_tar }}}{V_{\text {air_total }}} N_{\text {max }}
$$

where the initial number of pulses is $0, N_{\max }$ is the maximum number of pulses which can drive the piston of the air cylinder from one side to another. The number of pulses is the absolute value relative to the initial state.

Finally, the required number and frequency of pulses are produced to drive the motor and control the inner pressure of the device. We adopt the timer of the ARM processor to output the required frequency and corresponding number of pulses. The the number of output pulses is:

$$
N_{\text {offset }}=\operatorname{abs}\left(N_{\text {tar }}-N_{\text {cur }}\right)
$$

where $N_{\text {cur }}$ is the current absolute number of pulses. If $N_{\mathrm{tar}}-$ $N_{\text {cur }} \geq 0$, the stepper motor runs in clockwise direction; And if $N_{\text {tar }}-N_{\text {cur }}<0$, the stepper motor runs in counterclockwise direction. Therefore, the frequency of the output pulses can be defined as $f_{\text {pul }}=\frac{N_{\text {offset }}}{t_{\mathrm{d}}}$, however, the stepper motor is always constrained by its maximum start frequency $f_{\text {start }}$. If $f_{\text {pul }} \leq f_{\text {start }}$, we directly use the frequency $f_{\text {pul }}$ to drive the stepper motor. And if $f_{\text {pul }}>f_{\text {start }}$, we introduce a ladder method to accelerate the stepper motor to reach the target number of output pulses during $t_{\mathrm{d}}$. Then the target frequency $f_{\operatorname{tar}}$ satisfies following e- 


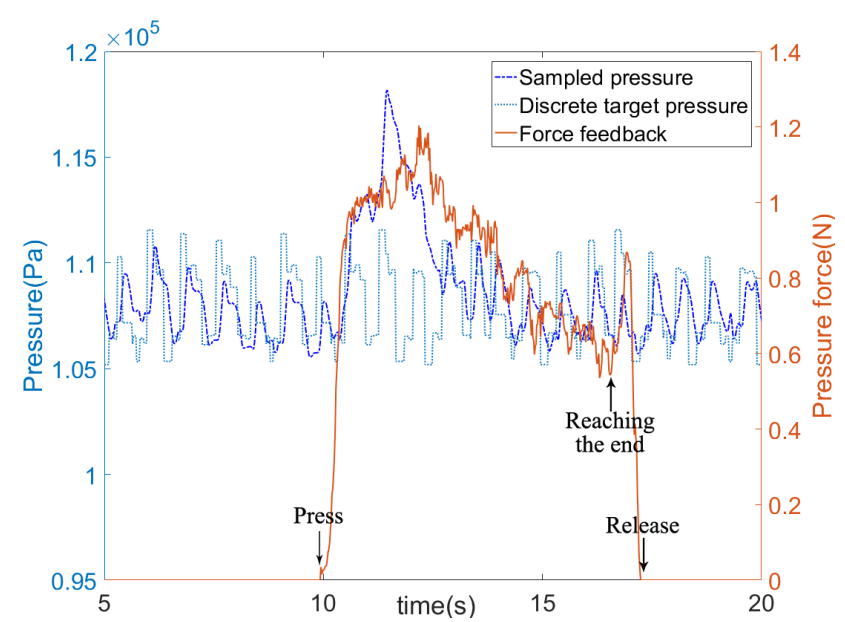

Fig. 3. The simulated coronary pressure and force feedback of the syringe device

quations and constraints:

$$
\left\{\begin{array}{l}
t_{1}=(i+1) t_{\mathrm{lad}} \\
t_{2}=\frac{N_{\mathrm{offset}}-s}{f_{\mathrm{i}+1}} \\
t_{\mathrm{d}}=t_{1}+t_{2} \\
s=(i+1) \frac{f_{\text {start }} t_{\mathrm{lad}}+f_{\mathrm{i}} t_{\mathrm{lad}}}{2} \\
f_{\mathrm{i}}=f_{\text {start }}+i \frac{f_{\mathrm{tar}}-f_{\mathrm{start}}}{N_{\mathrm{lad}}} \\
s<N_{\text {offset }} \\
f_{\text {start }} \leq f_{\mathrm{tar}} \leq f_{\mathrm{max}}
\end{array}\right.
$$

where $t_{1}$ is the time of the entire acceleration process, $t_{2}$ is the time of a constant speed process, $s$ is the number of output pulses in the acceleration process, $i \in\{0,1,2,3,4\}$ represents i-th acceleration ladder, $f_{\mathrm{i}}$ is the frequency of $\mathrm{i}$-th acceleration ladder, $N_{\text {lad }}=5$ is the maximum number of ladder, $t_{\text {lad }}=10 \mathrm{~ms}$ is the time of every acceleration ladder, $f_{\max }$ is the maximum target frequency. In this linear programming model equations, we compute $f_{\text {tar }}$ from $i=4$ to 0 and select the results that satisfy the given equations with the largest $i$ as the final target frequency.

\section{Experiments Results}

We have designed three experiments to assess the proposed syringe device. In Experiment 1, the plunger of the syringe was pushed to represent the injection of the contrast media. The simulated coronary pressure and its corresponding force feedback are analysed. In Experiment 2, the force feedback was computed with the coronary pressure and the force feedback without the coronary pressure. In Experiment 3, we integrated the syringe device with the simulation system for training an entire angiography diagnose procedure within a complex coronary artery simulation model.

\subsection{Experiment 1}

Fig. 3 shows the simulated results. The dark blue dot-dash curve represents the pressure sampled by the air pressure sensor; the light blue dotted curve shows the discrete target pressure; and the red solid curve is the force feedback of the plunger

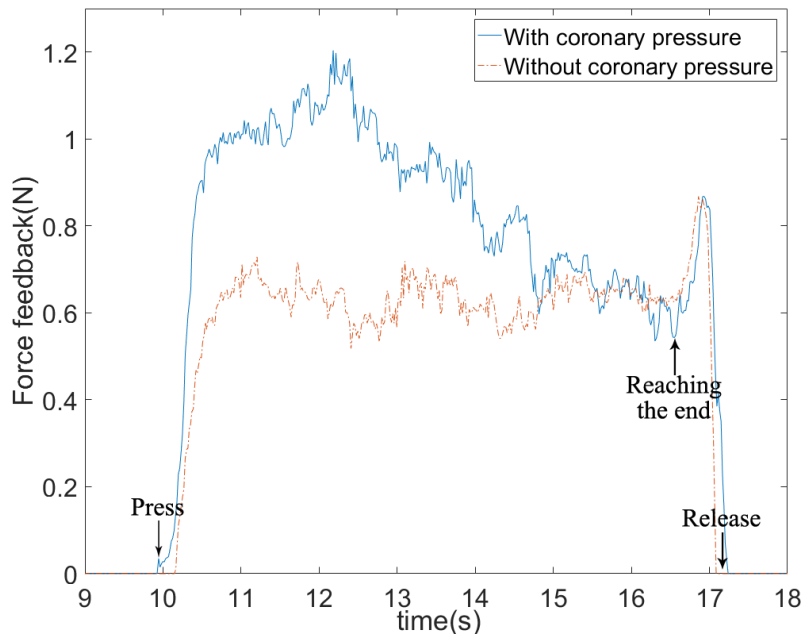

Fig. 4. Force feedback comparison

sampled by the force sensing resistor. The stepper motor runs to simulate coronary pressure at the begin. During this time the simulated pressure is very close to the target pressure. Then the plunger of the syringe pushes for the injection of the contrast media. The inner pressure of the device quickly increases to the peak, then decreases to the normal level; the force feedback increases rapidly at the beginning then decreases slowly until the plunger reaches the end of the syringe. During this period, the variation of the force feedback is almost simultaneous with the variation of pressure. When the plunger reaches the end of the syringe, the pressure force increases due to the pushing force and the reaction force from the end of syringe.

In this experiment, the lowest simulated pressures are stable within a range of level and not decrease with some rate, which indicates our syringe device is no loss of air. If our device exist leakage, the simulated pressure would decrease largely after we injected. So, this is a key aspect to guarantee the effectiveness of our device.

\subsection{Experiment 2}

Fig. 4 shows the force feedback of the proposed syringe device: the blue solid curve is the force feedback with coronary pressure and the red dot-dash curve is the force feedback without coronary pressure. The entire process is the same as the Experiment 1 . Due to the inner coronary pressure of the syringe device, the force feedback with the coronary pressure is much larger than the force feedback without the coronary pressure at the begin. When the plunger reaches the end of the syringe, these two extremum of force feedback are almost at the same level.

\subsection{Experiment 3}

The injection volume and the injection velocity of contrast media are the main influences to angiography simulation results. These data can automatically obtain from our device and init the physical simulation phase. Therefore, we designed the following experiment for achieving the diffusion effects of the contrast media by injecting the media using the syringe device. 


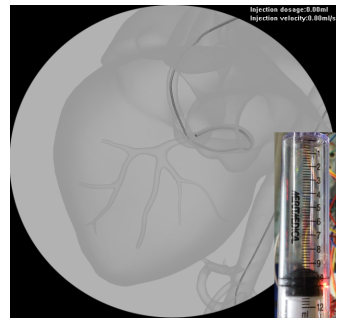

(a) $\mathrm{T}=0.00 \mathrm{~s}$

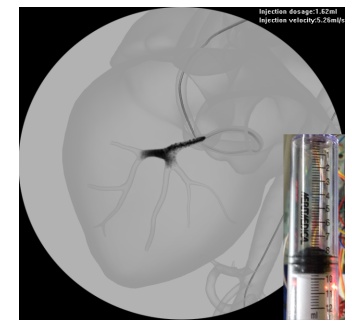

(b) $\mathrm{T}=0.23 \mathrm{~s}$

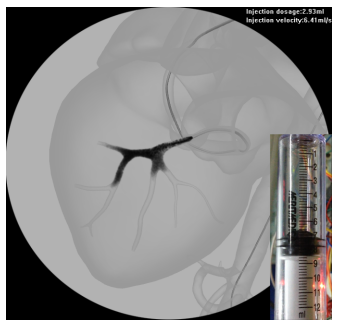

(c) $\mathrm{T}=0.42 \mathrm{~s}$

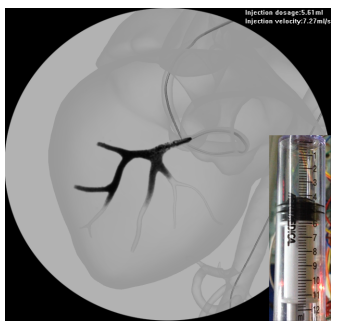

(d) $\mathrm{T}=0.83 \mathrm{~s}$

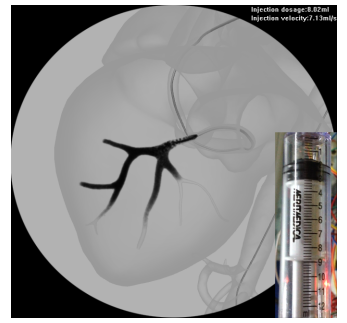

(e) $\mathrm{T}=1.12 \mathrm{~s}$

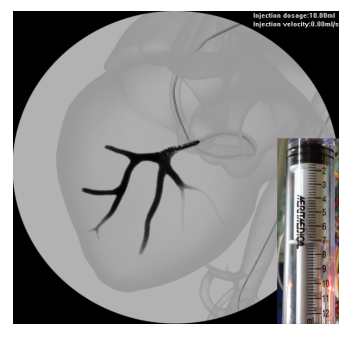

(f) $\mathrm{T}=1.51 \mathrm{~s}$

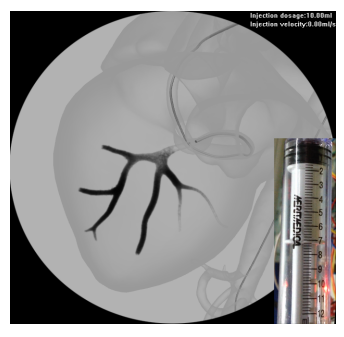

(g) $\mathrm{T}=2.08 \mathrm{~s}$

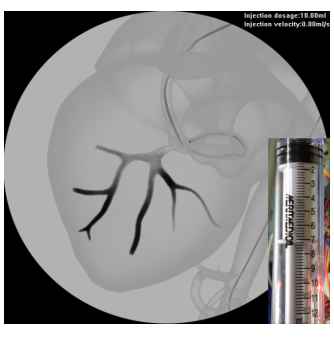

(h) $\mathrm{T}=2.59 \mathrm{~s}$

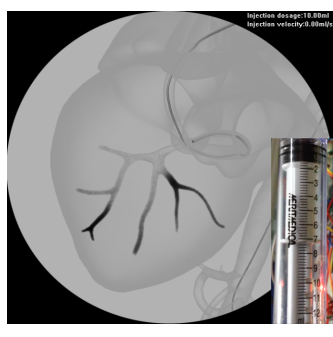

(i) $\mathrm{T}=\mathrm{T}=3.47 \mathrm{~s}$

Fig. 6. The rendering results of angiography

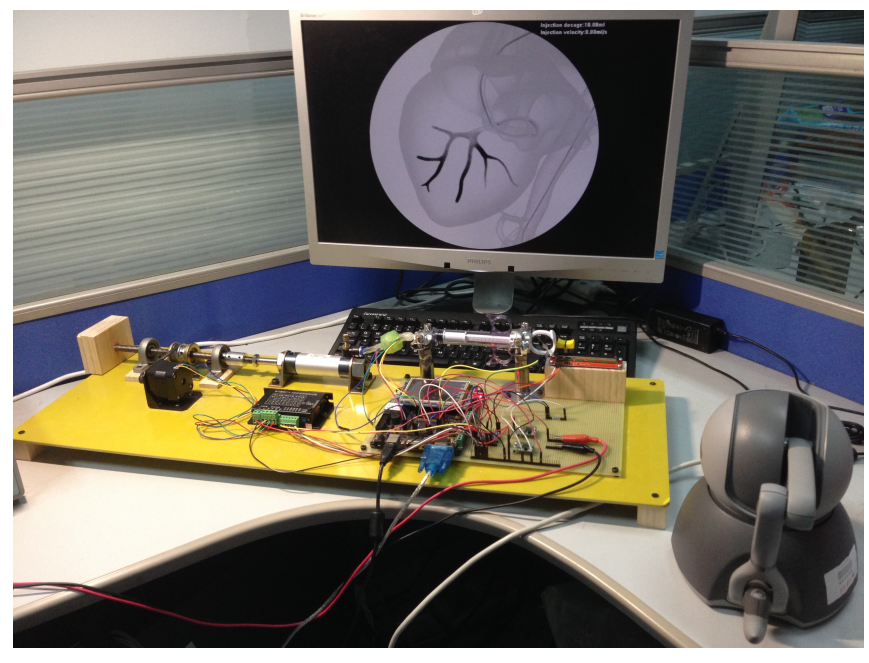

Fig. 5. Virtual angiography simulator

Fig. 5 shows the main component of virtual angiography simulator consisting of three components: a syringe device, a haptic device and a rendering system. Fig. 6 shows rendering results and the state of the syringe device at each time step from the contrast media injection, diffusion to disappearance.

- In Fig. 6(a), the catheter has reached the specified clinical location along the guidewire, and been prepared to push the piston to inject the contrast media. The dial of syringe device was at $10.00 \mathrm{ml}$ at this moment.

- In Fig. 6(b), the contrast media was at the beginning to be injected into the cardiovascular by the syringe device. At the time of $0.23 \mathrm{~s}$, the real injection dosage is $1.60 \mathrm{ml}$, and the estimated injection dosage and injection velocity were $1.62 \mathrm{ml}$ and $5.26 \mathrm{ml} / \mathrm{s}$ respectively with the injection dosage error as $+0.2 \%$.

- Fig. 6(c)(d)(e) show the cardiovascular developing process at every step while keeping the injection. The real injection dosage was $2.89 \mathrm{ml}, 5.58 \mathrm{ml}, 8.09 \mathrm{ml}$, respectively, and the estimated injection dosage was $2.93 \mathrm{ml}, 5.61 \mathrm{ml}, 8.02 \mathrm{ml}$ and the injection velocity was $6.41 \mathrm{ml} / \mathrm{s}, 7.27 \mathrm{ml} / \mathrm{s}, 7.13 \mathrm{ml} / \mathrm{s}$, respectively, the injection dosage error was $+0.4 \%,+0.3 \%,+0.7 \%$, respectively.

- Fig. 6(f) shows the contrast media diffuse condition after finishing injection. At the time of $1.51 \mathrm{~s}$, the real injection dosage was $10.00 \mathrm{ml}$, and the estimated injection dosage was $10.00 \mathrm{ml}$ and the injection velocity was $0.00 \mathrm{ml} / \mathrm{s}$.

- Fig. 6(g)(h)(i) show the diffusion and disappearance process of the contrast media under the push of the blood flow.

\section{Discussion}

The simulated pressure, in experiment 1 , approximates to the required objective pressure, and its pattern is also very similar to the real coronary pressure. Those are main factors to make a more realistic force feedback of injection. The tendency of force feedback and simulated pressure is consistent, which meats the expectation. However, the maximum of force not occurred at the same time with the maximum of simulated pressure. This situation may occur when the operator pushes the piston of syringe with lower injection speed near the maximum value of pressure. As you can see the force feedback result without simulation of coronary pressure in experiment2, the force also will change due to different injection speed.

To assess the integrated system in experiment 3 , we asked eleven surgeons with five or more years clinic experience in the field of interventional radiology from two hospitals to test our integrated virtual angiography simulator with the proposed haptic syringe device.

We designed a 20-point questionnaire and asked the medical experts to give subjective feedback. They were asked to rank statements on a seven-points Likert scale from 0 to 6 , where 0 is "very strongly disagree", 6 is "very strongly agree". The results 


\begin{tabular}{|l|l|}
\hline $\begin{array}{l}\text { The injection dosage and velocity of } \\
\text { contrast media is realistic. }\end{array}$ \\
\hline $\begin{array}{l}\text { The appearance of syringe device tool } \\
\text { looks real }\end{array}$ \\
\hline $\begin{array}{l}\text { The handfeel of operating the syringe } \\
\text { device tool is realistic }\end{array}$ \\
\hline $\begin{array}{l}\text { This simulator offers a user-friendly } \\
\text { learning environment }\end{array}$ \\
\hline $\begin{array}{l}\text { This simulator is effective for practicing } \\
\text { the basic skills of angiography }\end{array}$
\end{tabular}

Fig. 7. Average score of the assessment

of the expert feedback assessment are shown in Fig. 7, which presents boxplots. In boxplots, the light vertical bar presents the minimum and maximum scores of the corresponding question. The heavy vertical bar is the median and the darker and lighter boxes shows the lower and upper quartiles respectively.

In our simulator, we adopted fitting method to obtain the injection status with real injection dosage and velocity. Therefore, it provides a good realistic and accurate virtual injection performance for trainees and the median score of question 1 meets the expectation. Since we used the real physical control syringe as the main component of syringe device, seven experts scored 6 and four scored 5 in question 2. In the real angiography, the doctor needs to push the syringe hard during the injection due to the blood pressure. We adopted the stepper motor to control the inner air pressure to imitate the coronary pressure in order to produce a realistic force feedback of syringe. In this question, most surgeons agreed with the force feedback of our simulator.

However, the simulated coronary pressure is not fully realistic as the real heart coronary pressure and the long catheter to the coronary artery also will influence the force feedback of syringe. The expert assessment has shown that our system is well suited for training many interventional angiography procedures for clinical skills perpetration.

\section{Conclusion}

We have designed and implemented a novel interactive syringe device integrated with haptic feedback to be integrated into virtual angiography simulators for medical training. In order to increase the effectiveness of training, the new hardware device imitates the coronary pressure for force feedback of syringe and model the process of injecting radioactive contrast media. At last, the validity and efficacy of the simulator system and syringe device were assessed by medical doctors with good agreement and feedbacks.

However, there are still some limitations in our simulator, such as, existing sound noise of stepper motor. The influence of heart beating to angiography has not been considered in our current work. In the future, we will continue to improve the system to make a complete and practical virtual training simulator for endovascular interventional procedures. New psychomotor skill assessment for trainee could be also added into the simulation system.

\section{References}

[1] Cardiovascular diseases (cvds). May 17th, $2017 . \quad$ URL: http://www.who.int/news-room/fact-sheets/detail/ cardiovascular-diseases-(cvds)

[2] Bunce, N, Mohiaddin, R. An Atlas of Contrast-enhanced Angiography: Three-dimensional Magnetic Resonance Angiography. Taylor \& Francis; 2003.

[3] Hasnan, N, Engkasan, J, Husain, R, Davis, G. High-intensity virtual-reality arm plus fes-leg interval training in individuals with spinal cord injury. Biomedical Engineering/Biomedizinische Technik 2013;58(Supp.1):60-61.

[4] De Lazzari, C, Genuini, I, Pisanelli, DM, D’Ambrosi, A, Fedele, F. Interactive simulator for e-learning environments: a teaching software for health care professionals. Biomedical Engineering Online 2014;13(1):172:1-172:18.

[5] Brunberg, A, Heinke, S, Spillner, J, Autschbach, R, Abel, D, Leonhardt, S. Modeling and simulation of the cardiovascular system: a review of applications, methods, and potentials/modellierung und simulation des herz-kreislauf-systems: ein überblick zu anwendungen, methoden und perspektiven. Biomedizinische Technik/Biomedical Engineering 2009;54(5):233-244.

[6] Tang, W, Wan, T, Gould, D, How, T, John, W. A stable and real-time nonlinear elastic approach to simulating guidewire and catheter insertions based on cosserat rod. IEEE Transactions on Biomedical Engineering 2012;59(8):2211-2218.

[7] Westerdahl, DE. The necessity of high-fidelity simulation in cardiology training programs. Journal of the American College of Cardiology 2016;67(11):1375-1378.

[8] Voelker, W, Petri, N, Tönissen, C, Störk, S, Birkemeyer, R, Kaiser, E, et al. Does simulation-based training improve procedural skills of beginners in interventional cardiology? a stratified randomized study. Journal of interventional cardiology 2016;29(1):75-82.

[9] Tholey, G, Desai, JP, Castellanos, AE. Force feedback plays a significant role in minimally invasive surgery: results and analysis. Annals of surgery 2005;241(1):102-109.

[10] Coles, TR, Meglan, D, John, NW. The role of haptics in medical training simulators: A survey of the state of the art. IEEE Transactions on haptics 2011;4(1):51-66.

[11] van der Poorten, . Haptic feedback for medical applications, a survey. In: Proceedings of the 13th International Conference on New Actuators, Bremen, Germany. 2012, p. 18-21.

[12] Cotin, S, Dawson, S, Meglan, D, Shaffer, D, Ferrell, M, Bardsley, R, et al. Icts, an interventional cardiology training system. Studies in health technology and informatics 2000;70:59-65.

[13] Duriez, C, Cotin, S, Lenoir, J, Neumann, P. New approaches to catheter navigation for interventional radiology simulation. Computer Aided Surgery 2006;11(6):300-308.

[14] Tang, W, Lagadec, P, Gould, D, Wan, TR, Zhai, J, How, T. A realistic elastic rod model for real-time simulation of minimally invasive vascular interventions. The Visual Computer 2010;26(9):1157-1165.

[15] Huang, D, Tang, W, Wan, TR, John, NW, Gould, D, Ding, Y, et al A new approach to haptic rendering of guidewires for use in minimally invasive surgical simulation. Computer Animation and Virtual Worlds 2011;22(2-3):261-268. 
[16] Luboz, V, Zhang, Y, Johnson, S, Song, Y, Kilkenny, C, Hunt, C, et al. Imagine seldinger: first simulator for seldinger technique and angiography training. Computer Methods \& Programs in Biomedicine 2013;111(2):419-434.

[17] Wang, Y. Simulation of blood flow and contrast medium propagation for a vascular interventional radiology simulator. Master's thesis; Department of Computing: Imperial College London; 2009.

[18] Poyade, M, Lysakowski, A, Anderson, P. Development of a haptic training simulation for the administration of dental anaesthesia based upon accurate anatomical data. In: Conference and Exhibition of the European Association of Virtual and Augmented Reality. 2014, p. 143-147.

[19] Aggarwal, R, Black, S, Hance, J, Darzi, A, Cheshire, N. Virtual reality simulation training can improve inexperienced surgeons' endovascular skills. European journal of vascular and endovascular surgery 2006;31(6):588-593.

[20] Jensen, UJ, Jensen, J, Olivecrona, GK, Ahlberg, G, Tornvall, P. Technical skills assessment in a coronary angiography simulator for construct validation. Simulation in Healthcare: The Journal of the Society for Simulation in Healthcare 2013;8(5):324-328.

[21] Schimmel, DR, Sweis, R, Cohen, ER, Davidson, C, Wayne, DB. Targeting clinical outcomes: Endovascular simulation improves diagnostic coronary angiography skills. Catheterization and Cardiovascular Interventions 2016;87(3):383-388.

[22] Prenner, SB, Wayne, DB, Sweis, RN, Cohen, ER, Feinglass, JM, Schimmel, DR. Simulation-based education leads to decreased use of fluoroscopy in diagnostic coronary angiography. Catheterization and Cardiovascular Interventions 2018;91(6):1054-1059.

[23] Jan, GP, Fredrik, O. Interventional simulator control system. Jul. 23, 2013. URL: https://lens.org/089-323-998-746-629 uS 8491307 B2.

[24] Schuetz, M, Moenk, S, Vollmer, J, Kurz, S, Mollnau, H, Post, F, et al. High degree of realism in teaching percutaneous coronary interventions by combining a virtual reality trainer with a full scale patient simulator. Simulation in Healthcare Journal of the Society for Simulation in Healthcare 2008;3(3):242-246.

[25] Höfer, U, Langen, T, Nziki, J, Zeitler, F, Hesser, J, Müller, U, et al. Cathi-catheter instruction system. In: CARS 2002 Computer Assisted Radiology and Surgery. Springer; 2002, p. 101-106.

[26] Voelker, W. Computer simulation as training tool for coronary interventions. In: Catheter-Based Cardiovascular Interventions. Springer Berlin Heidelberg; 2013, p. 187-200.

[27] Wu, N, Tian, Y, Zou, X, Zhai, Y, Barringhaus, K, Wang, X. A miniature fiber optic blood pressure sensor and its application in in vivo blood pressure measurements of a swine model. Sensors \& Actuators B Chemical 2013;181(3):172-178. 